\title{
Study of Development of Non-Alcoholic Fatty Liver Disease (NAFLD) among Type-2 Diabetes Mellitus
}

\author{
Authors \\ Dr Shivani Bansal ${ }^{1}$, Dr Nilay Singh Rajput ${ }^{2}$ \\ ${ }^{1}$ Professor, Department of Medicine, Santosh Medical College \& Hospital \\ ${ }^{2}$ Post-Graduate $2^{\text {nd }}$ Year, Santosh Medical College \& Hospital
}

\section{Introduction}

Type 2 diabetes mellitus is a metabolic disorder of fuel homoeostasis characterized by hyperglycemia and altered lipid metabolism caused by islet $b$ cells being unable to secrete adequate insulin in response to varying degrees of over nutrition, inactivity, consequential overweight and insulin resistance.

Diabetes mellitus and lesser forms of glucose intolerance, particularly impaired glucose tolerance, can now be found in almost every population in the world and epidemiological evidence suggests that, without effective prevention and control programs, diabetes will likely to continue to increase globally. ${ }^{1}$

The prevalence of diabetes mellitusis growing rapidly worldwide and is reaching epidemic proportions. $^{2,3}$ It is estimated that there are currently 285 million people with diabetes worldwide and this number is set to increase to 438 million by year $2030 .{ }^{4}$ The major proportion of this increase will occur in developing countries of the world where disorder predominantly affects younger adults in the economically productive age group. ${ }^{5}$ There is also consensus that the South Asia region will include three of the top ten countries in the world (India, Pakistan and
Bangladesh) in terms of the estimated absolute numbers of people with diabetes ${ }^{3}$. Although the exact reasons why Asian Indians are more prone to type 2 diabetes at a younger age and premature cardiovascular disease (CVD) remains speculative, there is growing body of evidence to support concept of the "Asian Indian Phenotype". 6 This term refers to the peculiar metabolic features of Asian Indians characterized by a propensity to excess visceral adiposity, dyslipidemia with low HDL cholesterol, elevated serum triglycerides and increased small, dense LDL cholesterol, and an increased ethnic(possibly genetic) susceptibility to diabetes and premature coronary artery disease. ${ }^{6,7}$ Nonalcoholic fatty liver disease (NAFLD) is a global public health issue. This disease syndrome encompasses a spectrum of liver pathology, including steatosis, steatohepatitis, fibrosis and cirrhosis, and its incidence is increasing as the number of overweight and obese individuals in many countries increase. ${ }^{8,9}$ The prevalence of NAFLD is estimated to be approximately $75-92 \%$ in the morbidly obese population, $20 \%$ in the general population, and approximately $13-14 \%$ in the pediatric population. ${ }^{10}$ The prevalence of the progressive form, nonalcoholic steatohepatitis, is 
estimated to be $3-5 \%$, with $3-5 \%$ of those with the progressive form having advanced to cirrhosis. ${ }^{10}$ Patients with NAFLD were to have higher body mass index (BMI) values and waist circumferences than those without this syndrome, and metabolic syndrome was present in $61 \% .^{11}$ The detailed underlying mechanism and pathogenesis of NAFLD remain elusive, although the general overview is that two "hits" to the liver need to occur for this condition to progress to steatohepatitis: first, an accumulation of fat associated with insulin resistance, and second, activation of proinflammatory cytokines and stellate cells. ${ }^{12}$ Weight loss and increased exercise have been consistently associated with improvement in liver histology. Additional treatment is centered on attempts to manage the underlying metabolic risk factors. ${ }^{13}$

The health burden of NAFLD is expected to increase in future years due to aging population and to improved control of other major causes of chronic liver disease, such as hepatitis B and C, and alcoholic cirrhosis. ${ }^{14}$ Thus, the number of individuals at risk for end-stage liver disease and development of primary liver cancer is large. And NAFLD itself is an independent risk factor for cardiovascular disease, leads to increased allcause mortality, and also to increased liver-related mortality. ${ }^{10}$ A person is usually diagnosed with NAFLD after abnormal liver function tests prompt the use of either ultrasound or computed tomography to scan the liver for indications of fat infiltration. $^{10}$

Increase in endogenous glucose production, predominantly of hepatic origin are a major determinant of fasting hyperglycemia in type 2 diabetes. Lack of suppression of production after eating contributes to fed-state hyperglycemia. ${ }^{13}$ The mechanism underlying the dysregulation are complex, involving increased supply of gluconeogenic substrate from peripheral tissue, an effect of raised concentration of non-esterified fatty acids to activate hepatic gluconeogenesis, and the hepatic response to raised concentration of glucagon. ${ }^{15,16}$ Type 2 diabetes is strongly associated with Non-Alcoholic Fatty Liver Disease each is highly predictive of the other. ${ }^{15,16}$ The majority of patients diagnosed with NAFLD are asymptomatic. ${ }^{17,19}$ When present, clinical symptoms and physical findings are nonspecific and unreliable for diagnosing and assessing disease severity in patients with NAFLD. Patients might have hepatomegaly, general malaise, abdominal discomfort, vague right upper quadrant pain, nausea, and other nonspecific symptoms referred to gastrointestinal tract. Clinical examination may reveal ascites, splenomegaly, spider angiomas, palmer erythema, caput medusa, and jaundice in small percentage of patients who present with NASH related cirrhosis. ${ }^{17,19}$ The features more consistently found to be associated with disease severity include obesity, older age, diabetes, and hypertension. ${ }^{18}$

The ratio of AST/ALT is usually less than 1 in patients who have either no or minimal fibrosis, although this ratio may be greater than 1 with development of cirrhosis. ${ }^{20}$ Gammaglutamyltransferase (GGT) in the serum is frequently elevated in patients with NAFLD, and it has been reported to be associated with increased mortality. ${ }^{21,22}$

The NAFLD liver fat score includes, as variables, the presence of metabolic syndrome and T2DM, fasting serum insulin, serum AST, and the AST/ALT ratio. Bedogni et al developed the fatty liver index (FLI), which uses the body mass index (BMI), waist circumference, triglyceride level, and GGT in the general population with low prevalence of T2DM. This index varies from 0 to 100 to detect liver steatosis. ${ }^{23}$

The HAIR (Hypertension, ALT and Insulin Resistance) score was designed to predict a NASH diagnosis, and includes a combination of the presence of hypertension, elevated ALT, and insulin resistance. The presence of at least 2parameters predicted NASH with both a high sensitivity and specificity. $^{24}$ Palekar et al generated a clinical model to distinguish NASH from simple steatosis by combining 6 different 
variables including age, gender, AST, BMI, the AST/ALT ratio and serum $\mathrm{HA}^{25}$

Although many imaging tools have been assessed in NAFLD subjects, their main focus has been the quantification of liver fat. The results of these imaging tests cannot be used to differentiate between the histological subtypes of simple steatosis or NASH, nor can they be used to stage the degree of fibrosis. ${ }^{26,27}$ In this section, each imaging modality is explained, while referring to the detection of hepatosteatosis, steatohepatitis, and fibrosis.

USG is currently the most common method for screening asymptomatic patients with elevated liver enzymes and suspected NAFLD. USG findings of fatty liver include hepatomegaly, diffuse increase in the echogenicity of the liver parenchyma, and vascular blunting. Non steatotic hepatic parenchyma exhibits an echotexture similar to that of renal parenchyma, but becomes "brighter" when infiltrated with fat. ${ }^{28}$ This hepatorenal contrast can be used for detecting hepatosteatosis. ${ }^{28,29}$

Computed Tomography allows for a more quantitative assessment with measurement of liver attenuation in Hounsfield units (HUs) compared to USG, but the information about liver attenuation is not uniform when reported by radiologists. Magnetic Resonance Imaging (MRI) and Proton Magnetic Resonance Spectroscopy (MRS)-A good correlation has been reported between MRI, USG and histology. Transient Elastography is a non-invasive method of assessing liver fibrosis which uses ultrasound based technology to measure liver stiffness. It is less validated in NAFLD but combination with one or more serum marker panels might be a potential approach for the non-invasive measurement of fibrosis in NAFLD. Liver biopsy is the gold standard for diagnosis and has an additional benefit of distinguishing between NASH and simple steatosis but has got its limitations as it is a invasive procedure.

NAFLD and type 2 diabetes mellitus frequently coexist because they share the risk factors of excess adiposity and insulin resistance. The prevalence of $\mathrm{T} 2 \mathrm{DM}$ or impaired fasting glucose ranges from !8-33\% in patients with NAFLD, whereas it ranges from $49-62 \%$ in T2DM patients who have NAFLD ${ }^{30,31,32,33}$ Gupte et al $^{33}$ in 2004 found that mild, moderate and severe NAFLD was present in $65.5 \%, 12.5 \%$ and $9.35 \%$ of otherwise asymptomatic T2DM respectively

\section{Material and Methods \\ Study Design}

We carried out a cross-sectional Case Control study on type-2 diabetic patients and general population respectively attending outpatient department of General Medicine, Santosh Medical College and Hospital, Ghaziabad.

A total number of 150 cases of type- 2 diabetes mellitus population and 50 controls of general healthy population were recruited in present study strictly by outlined inclusion and exclusion criteria. An informed consent was taken from all the cases and controls before their inclusion into study. The present study was approved by ethics committee of Santosh Medical College and Hospital, Ghaziabad.

All 150 cases and 50 controls underwent Ultrasonography of liver to diagnose NAFLD by non-invasive means. NAFLD is defined as any degree of fatty liver in the absence of alcohol intake. NAFLD, if present, was classified based on standard Ultrasonography criteria as:

Grade 1 (Mild Steatosis): slightly increase liver echogenicity with normal vessels and absent posterior attenuation

Grade 2 (Moderate steatosis): moderately increase liver echogenicity with partial dimming of vessels and early posterior attenuation

Grade 3 (Severe Steatosis): diffusely increased liver echogenicity with absence of visible vessels and heavy posterior attenuation.

Based on above means of diagnosing presence/absence of NAFLD, Cases and Controls were further subdivided into 4 Groups of NAFLD cases and controls and Non-NAFLD cases and controls respectively. 


\section{Inclusion Criteria for Cases and Controls}

$>$ All Cases and Controls were taken in between age of 31-60 years of both genders.

$>$ Adults who self-report in out-patients dept, or who are screened and found to have diabetes were included as Cases.

$>$ Diabetic Patients.

$>$ All controls underwent screening for diabetes as per criteria laid down in inclusion criteria.

$>$ The controls were invited to voluntarily participate and informed consent was obtained for USG, analytical tests and performing screening test for diabetes to ensure that controls are Non-Diabetics.

\section{Exclusion Criteria for Cases and Controls:}

Alcohol intake>30g/day in males and $>20 \mathrm{~g} /$ day in females.

Presence of chronic liver disease like cholelithiasis, hydatid cyst, haemochromatosis, glycogen storage disease, abetalipoprotenemia and others.

$>$ Presence of the hepatitis B virus surface antigen or the presence of virus hepatitis $\mathrm{C}$ antibodies.

Subjects who are exposed to drugs or toxins like ethylbromide, estrogens, glucocorticoids, highly active antiretroviral therapy, barium salts, chromates, phosphorous, thallium, and uranium compounds, amiodarone, tamoxifen and others.

> Subjects with conditions or disease hindering data collection and follow up of the study such as incapacitating diseases, cognitive deterioration, institutionalized patients or subjects with no fixed address in any of the basic areas of the study.

$>$ Subjects who do not provide written informed consent to participate in the study.

$>$ Study having pre-existing fatty liver disease will be excluded.

\section{Data Collection}

$>$ The cases and controls with any exclusion criteria in their clinical history were not invited to participate in the study.

$>$ Detailed history of both controls and cases were taken regarding any presenting complaint and detailed physical examination (i.e Height, Weight, BMI, Waist Circumference, Hip Circumference) should be done.

$>$ Blood pressure was measured, and ECG was done

> Laboratory tests done including Hemogram, Serum Creatinine with special reference to lipid profile, Fasting \& Post Prandial Sugar levels, HbA1c estimation after about 8 hours of overnight fasting, venous samples were taken from all the cases and controls.

\section{Imaging of abdomen by ultrasonography}

All patients underwent ultrasound (USG) of the abdomen to detect fatty changes in the liver. The scanning was done for an average of 30 mins and images obtained were recorded. Fatty liver was defined as the presence of an ultrasonography pattern consistent with "bright liver" with evident ultrasonography contrast between hepatic and renal parenchyma, vessel blurring, and narrowing of the lumen of the hepatic veins in the absence of findings suggestive of chronic liver disease. All cases and controls were stratified by absence and presence of NAFLD by USG into 4 groups as described above. Grading of severity of NAFLD was done as per standard criteria described above in materials and methods.

\section{Statistical Analysis}

Statistical analysis were done using t-test and chisquare test using standard SPSS version as appropriate. Univariate and Multivariate analysis were done by using logistic regression model. Multivariate analysis were done by ENTER method. 
Observations and Results

Based on presence/absence of NAFLD, Cases and Controls were subdivided into 4 Groups:-

Group 1: Cases: Diabetic population of cases having NAFLD on USG $(\mathrm{N}=91)$

Group 2: Cases: Diabetic population of cases NOT having NAFLD on USG (N=59)

Group 3: Controls: General healthy population of control having NAFLD on USG (N=9)

Group 4: Controls: General healthy population of control NOT having NAFLD on USG $(\mathrm{N}=41)$
Distribution of Gender of Cases (Diabetic population) Study Groups as per Presence/ Absence of NAFLD (Prevalence)

\begin{tabular}{|l|c|c|c|}
\hline S.No & Gender & \multicolumn{2}{|c|}{ Cases(n=150) } \\
\hline & & Group1N(\%age) & Group2N(\%age) \\
\hline 1 & Males & $46(30.66 \%)$ & $28(18.67 \%)$ \\
\hline 2 & Females & $45(30 \%)$ & $31(20.67 \%)$ \\
\hline 3 & Total(M+F) & $91(60.66 \%)$ & $59(39.34 \%)$ \\
\hline
\end{tabular}

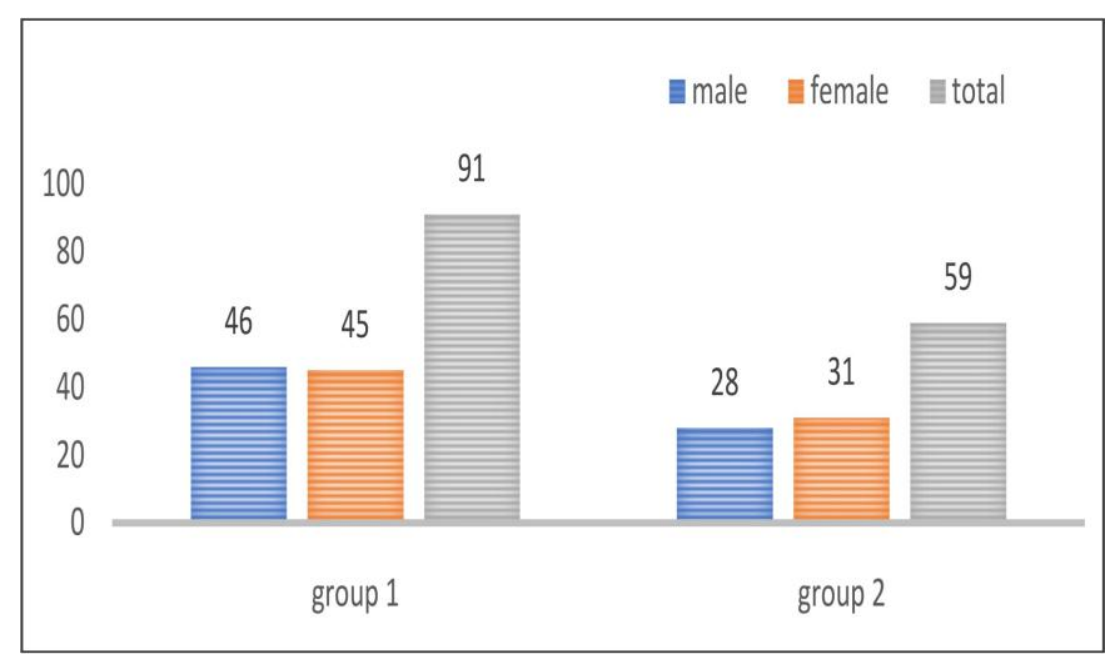

In this study of diabetic population, the prevalence of NAFLD is found to be $60.66 \%$.

Distribution of NAFLD among Cases and Controls of group 1 and 3 respectively, as per Grading (Severity) of NAFLD on basis of criteria laid down for USG of Liver in material and methods.

\begin{tabular}{|l|c|c|c|c|}
\hline S.No & $\begin{array}{c}\text { Grading of NAFLD } \\
\text { (USG Criteria) }\end{array}$ & $\begin{array}{c}\text { Diabetic Cases } \\
\mathrm{N}=91\end{array}$ & $\begin{array}{c}\text { Non-Diabetic } \\
\text { Controls N=9 }\end{array}$ & $\begin{array}{c}\text { Total } \\
\text { N=100 }\end{array}$ \\
\hline 1 & Grade 1 & 44 & 6 & $50(50 \%)$ \\
\hline 2 & Grade 2 & 36 & 3 & $39(39 \%)$ \\
\hline 3 & Grade 3 & 11 & 0 & $11(11 \%)$ \\
\hline & Total & 91 & 9 & 100 \\
\hline
\end{tabular}

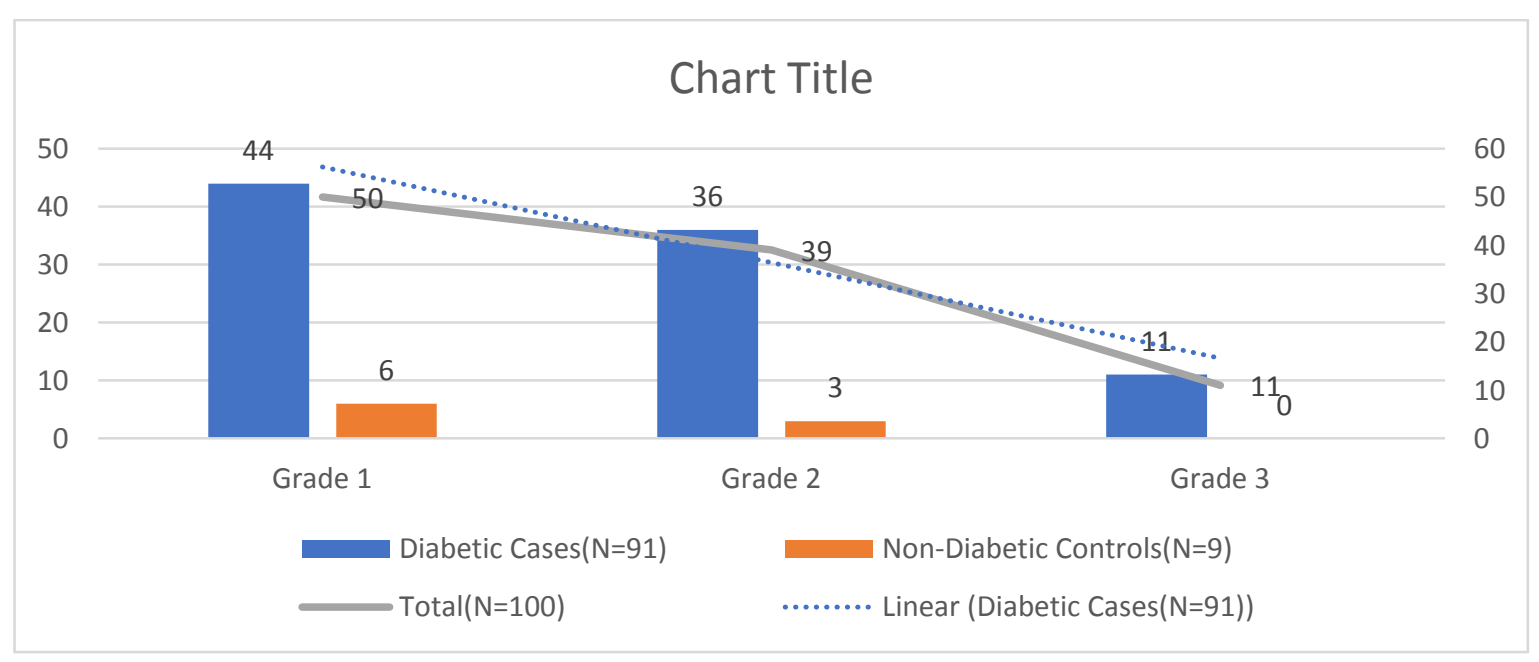


While performing ultrasonography both group 1 cases and group 3 controls in present study, subjects found to have NAFLD were stratified into 3 grades of fatty liver as per criteria laid down. In group 1, Grade 1 NAFLD was more prevalent i.e. 44(48.35\%) cases, Grade 2 NAFLD with prevalence of $36(39.56 \%)$ cases. Only $11(12.80 \%)$ were found to have NAFLD of grade 3 in group 1 . In group $3,2 / 3^{\text {rd }}$ of controls i.e. $6(66.67 \%)$ out of 9 having NAFLD had grade 1 NAFLD and rest having grade 2 (33.33\%). In control group, there was no subject was found to have grade 3 NAFLD.

Association of Age and Anthropometric Parameters as Risk Factors for NAFLD among Cases and Controls study groups (Diabetic population)

\begin{tabular}{|l|c|c|c|}
\hline Variables & Cases & Control & P value \\
\hline Age(years) & $46.5(7.2)$ & $45.4(7.2)$ & 0.520 \\
\hline Weight(kgs) & $76.2(8.8)$ & $65.7(11.8)$ & $<0.001^{*}$ \\
\hline BMI(kg/m2) & $29.9(2.9)$ & $28.2(4.9)$ & $0.014^{*}$ \\
\hline $\begin{array}{l}\text { Waist } \\
\text { circumference(cms) }\end{array}$ & $85(5)$ & $74(3.9)$ & $<0.001^{*}$ \\
\hline Waist Hip ratio & $0.9(.1)$ & $0.7(0)$ & $<0.001^{*}$
\end{tabular}

Association of Major laboratory findings as Risk Factors for NAFLD among Cases and Controls study groups (Diabetic population)

\begin{tabular}{|l|c|c|c|}
\hline Variables & Cases & Control & P value \\
\hline FBS & $146.2(25.1)$ & $81.4(8.9)$ & $<0.001^{*}$ \\
\hline PPBS & $239.7(41)$ & $152.2(28.6)$ & $<0.001^{*}$ \\
\hline Hb1Ac & $7.1(1.1)$ & $5.6(0.4)$ & $<0.001^{*}$ \\
\hline AST & $46.6(25.5)$ & $63.3(27.7)$ & 0.07 \\
\hline ALT & $100(37.7)$ & $46.2(26.1)$ & $<0.001^{*}$ \\
\hline Total & $219.7(65.1)$ & $184.1(62.7)$ & $0.001^{*}$ \\
\hline Cholesterol & $39.7(7.7)$ & $44.1(6.7)$ & $0.002^{*}$ \\
\hline HDL & $186.7(49.1)$ & $155(40.8)$ & $<0.001^{*}$ \\
\hline LDL & $253.6(68.8)$ & $149.9(56.1)$ & $<0.001^{*}$ \\
\hline TG & & &
\end{tabular}

Association of Systolic and Diastolic blood pressure as Risk Factors for NAFLD among Cases and Controls study groups (Diabetic population)

\begin{tabular}{|l|c|c|c|}
\hline $\begin{array}{l}\text { Blood } \\
\text { Pressure }\end{array}$ & $\begin{array}{c}\text { Group 1 } \\
\text { N=91 }\end{array}$ & $\begin{array}{c}\text { Group 2 } \\
\text { N=59 }\end{array}$ & P value* \\
\hline Systolic & $129.53 \pm 6.96$ & $125.67 \pm 5.75$ & $0.001^{*}$ \\
\hline Diastolic & $84.51 \pm 6.17$ & $80.71 \pm 5.71$ & $0.000^{*}$ \\
\hline
\end{tabular}

Association of Duration of Diabetes as Risk Factors for NAFLD among Cases study groups (Diabetic population)

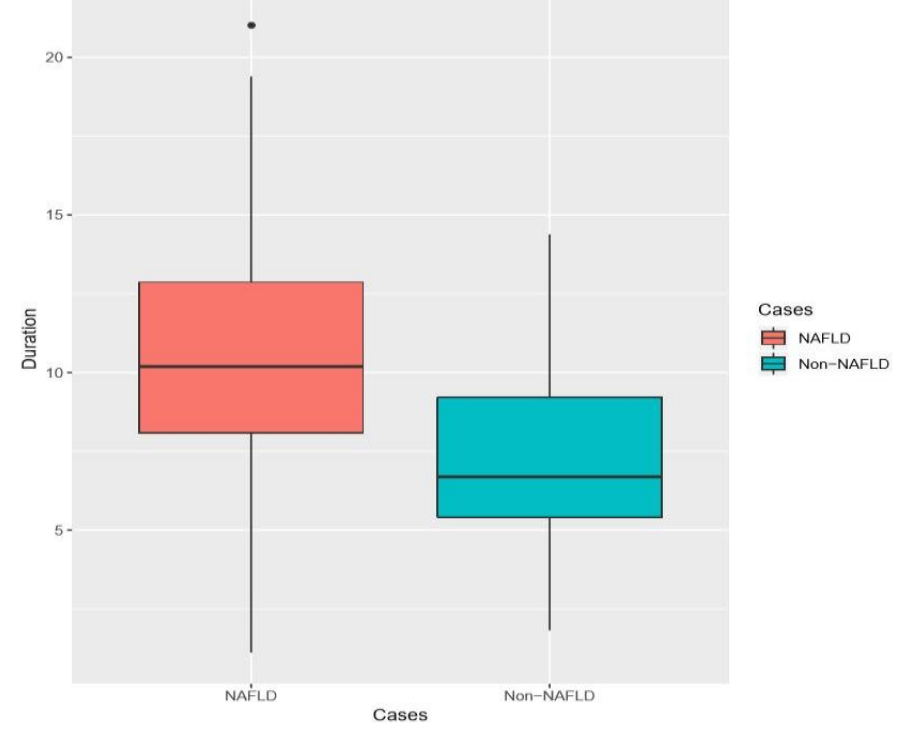

On comparing average duration of Diabetes, in group 1 and 2, present study found significant association of duration of diabetes in years and development of NAFLD with P value of 0.000 . Mean duration of diabetes in group 1 and 2 was $10.26 \pm 3.83$ and $7.14 \pm 4.62$ years respectively.

Regression analysis to determine the risk factors for NAFLD in the total study population

\begin{tabular}{|c|c|c|c|}
\hline S.no & Variables & \multicolumn{2}{|c|}{ Overall study population } \\
\hline & & $\begin{array}{l}\text { Univariate } \\
\text { Analysis }\end{array}$ & $\begin{array}{c}\text { Multivariate } \\
\text { Analysis }\end{array}$ \\
\hline 1 & $\operatorname{Sex}(M / F)$ & 0.572 & 0.168 \\
\hline 2 & BMI (nor/o.w) & $0.001 *$ & 0.855 \\
\hline 3 & SBP (>130 mmhg) & $0.000 *$ & $0.003 *$ \\
\hline 4 & DBP (>85 mmhg) & $0.000 *$ & $0.002 *$ \\
\hline 5 & T. Chl (>200 mg/dl) & $0.000 *$ & 0.153 \\
\hline 6 & $\begin{array}{c}\text { HDL }(<40 \&<50 \mathrm{mg} / \mathrm{dl} \\
\text { males and females })\end{array}$ & $0.002 *$ & 0.962 \\
\hline 7 & TG $(>150 \mathrm{mg} / \mathrm{dl})$ & $0.000 *$ & $0.011 *$ \\
\hline 8 & $\mathrm{AST}(>40 \mathrm{IU} / \mathrm{L})$ & 0.572 & 0.962 \\
\hline 9 & ALT (>40 IU/L) & $0.000 *$ & $0.043 *$ \\
\hline 10 & PPBS (>200 mg/dl) & $0.000 *$ & 0.478 \\
\hline 11 & $\operatorname{HbA} 1 \mathrm{C}(>7 \%)$ & $0.000 *$ & 0.161 \\
\hline 12 & FBS (>126 mg/dl) & $0.000 *$ & 0.672 \\
\hline 13 & $\begin{array}{c}\text { Family history of } \\
\text { (present/absent) } \\
\text { (CAD, DM, SMK) }\end{array}$ & 0.247 & 0.521 \\
\hline
\end{tabular}

In present study, we took whole population of cases and controls $(\mathrm{N}=200)$ and compared risk factors by regression analysis for development of 
NAFLD irrespective of diabetes status of both Cases and Controls Population.

On Univariate regression analysis, High BMI, SBP, DBP, TG, T.Chl, FBS, PPBS, ALT, HbA1c and low value of HDL were found to play a significant role in development of NAFLD independently, irrespective of diabetes whereas on Multivariate regression analysis, only High, SBP, DBP, TG and ALT levels played significant role in development of NAFLD irrespective of diabetes.

\section{Discussion}

As per increasing prevalence of type $2 \mathrm{DM}$ in India and suspected to be having every fifth diabetic in world as Indian by 2030, studies on prevalence of NAFLD in Type-2 DM patients are very less.

Viswanathan et al ${ }^{34}$ in 2010 conducted study on 2161 diabetic subjects in southern India and found prevalence of NAFLD $7.1 \%$ on ultrasonography. Ferreira et $\mathrm{al}^{35}$ in 2010 studied 78 subjects of type 2 diabetes mellitus and found prevalence of $42 \%$ of NAFLD in them by means of ultrasonography.

The present study found to have BMI, Weight, WC and WHR to be significantly associated with development of NAFLD in type 2 diabetic population. Cheng chungfu et $\mathrm{al}^{36}$ in 2009 also found ALT levels to be significantly associated with development of NAFLD in general population. The present study found to have both systolic and diastolic blood pressure being important risk factor in developing NAFLD in type 2 diabetic patients. There was strong association between Triglycerides level and development of NAFLD.

On comparing family history of CAD, HTN and DM in diabetic population of cases present study did not found any significant association between these variables and development of NAFLD. History of smoking was also found to be insignificant in development of NAFLD in diabetic population. Whereas on comparing average duration of diabetes, in group 1 and 2 , present study found significant association of duration of diabetes in years and development of NAFLD with $P$ value of 0.000 . Mean duration of diabetes in group 1 and 2 was $10.25 \pm 3.83$ and $7.14 \pm 4.62$ years respectively. This implies that development of NAFLD is significantly associated with presence of diabetes in given population and diabetes predisposes to development of NAFLD in diabetic population. As duration of diabetes increases in given population, there is increased risk of development of NAFLD in diabetic population.

On comparing family history of CAD, Hypertension and Diabetes mellitus in between group 3 and 4, present study did not found any of these variables significant for development of NAFLD ( $\mathrm{p}=0.103,0.158$ and 0.255 ). Smoking too was not significant in development of NAFLD in general population of controls.

\section{Conclusion}

It is observed that prevalence of NAFLD in type-2 diabetic population is $40 \%$ more as compared to same age group of general (non-diabetic) population. Combination of markers like TG, ALT, HbA1C, Blood Pressure, BMI, WC, WHR and screening by Ultrasonography of liver can act as group of non-invasion markers predicting the prevalence of NAFLD in diabetic population.

\section{Bibliography}

1. World Health Organization. Prevention of diabetes mellitus. Technical Report Series no. 844.Geneva: World Health Organization, 1994.

2. King H, Rewers M. Diabetes in adults is now a Third World problem. The WHO Ad Hoc Diabetes Reporting Group. Bull World Health Organ 1991;69:643-8.

3. Bjork S, Kapur A, King H, Nair J, Ramachandran A.2.Global policy: aspects of diabetes in India. Health Policy 2003;66:61-72

4. Sicree R,Shaw J, Zimmet P. Diabetes and impaired glucose tolerance. In: Gan D, editor. Diabetes atlas. International 
Diabetes Federation. Belgium:

International Diabetes Federation; 2009. (4th ed)p.1-105.

5. Mather HM, Verma NP, Mehta SP, Madhu $\mathrm{SV}$, Keen H. The Prevalence of known diabetes in Indians in New Delhi and London. J Med Assos Thai 1987;70:54-8.

6. Deepa R, Sandeep S, Mohan V. Abdominal obesity, visceral fat 5.and Type 2 diabetes- "Asian Indian Phenotype". In: Mohan V, Gundu HR Rao, editors. Type 2 diabetes in South Asians: Epidemiology, risk factors and prevention. New Delhi: Jaypee Brothers Medical Publishers; 2006.p.138-52

7. Joshi R. Metabolic syndrome - Emerging clusters of the Indian Phenotype. J Assoc Physicians India2003;51:445-6.

8. Yan E, Durazo F, Tong M, Hong K. Nonalcoholic fatty liver disease: pathogenesis, identification, progression, and management. Nutr Rev 2007;65 (8):376-84.

9. Kallwitz ER, Kumar M, Aggarwal R, Berger R, Layden Almer J, Gupta N, Cotler SJ, Ethnicity and nonalcoholic fatty liver disease in an obesity clinic: the impact of triglycerides, Dig Dis Sci 2008;53:1358-63.

10. Erickson SK. Nonalcoholic fatty liver disease, J Lipid Res 2009:50(Suppl.): S412-6. Epub; 2008Dec 12.

11. Radu C, Grigorescu M, Crisan D, Lupsor M, Constantin D, Dina L Prevalence and associated risk factors of non-alcoholic fatty liver disease in hospitalized patients. J Gastrointestin Liver Dis. 2008; 17(3):255-60.

12. Chen $\mathrm{CH}$, Huang MH, Yang JC,Nien CK, Yang CC, Yeh YH, Yueh SK, Prevalence and risk factors of nonalcoholic fatty liver disease in an adult population in Taiwan: metabolic significance of nonalcoholic fatty liver disease in non obese adults, $\mathrm{J}$ Clin Gastroenterol 2006;40(8):745-52.
13. Cheung O, Sanyal AJ. Recent advances in nonalcoholic fatty liver disease, Curr Opin Gastroenterol 2009; 25(3):230-7.

14. Lazo M, Clark JM. The epidemiology of nonalcoholic fatty liver disease:a global perspective. Semin Liver Dis 2008;28(4):339-50,

15. Shah P, Basu A, Rizza R. Fat-induced liver insulin resistance. Curr Diabetes Rep. 2003;3:214-18.

16. Nolan CJ. Failure of islet $\beta$-cell compensation for insulin resistanct causes type 2 diabetes: what causes NAFLD and NASH? J Gastroenterol1 Hepatol. 2010;25:1591-97.

17. Lewis JR, Mohanty SR. Nonalcoholic fatty liver disease: a review and update. Digestive Diseases and Sciences.2010; 55(3):560-78.

18. Angulo P. Medical progress: nonalcoholic fatty liver disease. The New England Journal of Medicine. 2002; 346(16):Z22131.

19. Smith BW and Adams LA. Nonalcoholic fatty liver disease and diabetes mellitus: pathogenesis and treatment. Nature Reviews Endocrinology. 2011;7(8): 45665.

20. Angulo P, Keach JC, Batts KP, Lindor KD. Independent predictors of liver fibrosis in patients witj nonalcoholic steatohepatitis. Hepatology. 1999; 30(6): 1356-62.

21. R Haring, H. Wallaschofski, M. Nauck, S.E. Baumeister, H. V"olzke, "Ultrasonographic hepatic steatosis increases prediction of mortality risk from elevated serum gamma-glutamyltranspeptidase levels," Hepatology. 2009; vol. 50; no. 5:pp. 1403-11.

22. Ruhl CE, Everhart JE. Elevated serum alanine aminotransferase and gammaglutamyltransferase and mortality in the United States population. Gastroenterology. 2009;136(2): 477-85. 
23. Bedogni G, Bellentani S, Miglioli L. The fatty liver index:a simple and accurate predictor of hepatic steatosis in the general population. BMC Gastroenterology 2006;33.

24. Dixon J. Bhathal P, O'Brien P. Nonalcoholic fatty liver disease: predictors of nonalcoholic steatohepatitis and liver fibrosis in the severely obese. Gastroenterology 2001;121(1):91-100

25. Ratziu V, Massard J, Charlotte F. Diagnostic value of biochemical markers (Fibro Test-Fibro SURE) for the prediction of liver fibrosis in patients with nonalcoholic fatty liver disease. BMC Gastroenterology 2006;6.

26. Saadeh S, Younossi Z, Remer E. The utility of radiological imaging in nonalcoholic fatty liver disease. Gastroenterology 2002;123(3):745-750.

27. J.A. Marrero, R.J. Fontana, G.L. Su, Conjeevaram H, Emick D, Lok S.NAFLD may be a common underlying liver disease in patients with hepatocellular carcinoma in the United States. Hepatology 2002;36(6):1349-1352.

28. Osawa H, Y. Mori Y. Sonographic diagnosis of fatty liver using a histogram technique that compares liver and renal cortical echo amplitudes. Journal of Clinical Ultrasound 1996;24(1):25-29.

29. Sanyal A.AGA technical review on nonalcoholic fatty liver disease. Gastroenterology 2002;123(5):1705-1725.

30. Browning J, Szczepaniak L, Dobbins R. Prevalence of hepatic steatosis in an urban population in the united states: impact of ethnicity. Hepatology 2004; 40(6): 13871395.

31. Fan J, Zhu J, Li XJ. Prevalence of and risk factors for fatty liver in a general population of Shanghai, China. Journal of Hepatology 2005;(3):508-514.
32. Jimba S, Nakagami T, Takahashi $M$. Prevalence of non-alcoholic fatty liver disease and Itsassociation with impaired glucose metabolism in Japanese adults. Diabetic Medicine 2005;22(9):1141-1145.

33. Gupte P, Amarapurkar D, Agal S. Nonalcoholic steatohepatitis in type 2 diabetes mellitus. Journal of Gastroenterology and Hepatology 2004;19(8):854-858.

34. Vishwanathan V, Mahesh K. Association of non-alcoholic fatty liver disease with diabetic microvascular and macrovascular complications in south Indian subjects. International journal of diabetes in developing countries 2010;4.

35. Vera S, Ferreira G, Pernambuco B. Frequency and risk factors associated with non-alcoholic fatty liver disease in patients with type 2 diabetes mellitus. Arq Bras Endocrinol Metab.2010;54(4).

36. Chung $\mathrm{Fu} \mathrm{C}$, Chen M. The Risk Factors for Ultrasound-diagnosed Non-alcoholic Fatty Liver Disease among Adolescents. Ann Acad Med Singapore 2009;(38):1521. 